\title{
Current Diagnosis and Management of Gastrojejunocolic Fistula
}

\author{
Can Kece ${ }^{\mathrm{a}}$ Tahsin Dalgic ${ }^{\mathrm{a}}$ Isılay Nadir ${ }^{\mathrm{b}}$ Behlul Baydar \\ Gurel Nessar $^{\mathrm{a}}$ Burhan Ozdil ${ }^{c} \quad$ E. Birol Bostanci ${ }^{\mathrm{a}}$ \\ Departments of a Gastroenterologic Surgery Clinic and ${ }^{\text {b } G a s t r o e n t e r o l o g y ~ C l i n i c, ~}$ \\ Yuksek Ihtisas Hospital, Ankara, and 'Department of Gastroenterology Clinic, \\ Numune Research Hospital, Trabzon, Turkey
}

\section{Key Words}

Gastrojejunocolic fistula - Gastrectomy - Gastrocolic fistula - Recurrent peptic ulcer

\begin{abstract}
We herein report the case of a 51-year-old man with gastrojejunocolic fistula. It is one of the late severe complications of gastrectomy and gastrojejunostomy and is considered to be induced by a stomal ulcer due to inadequate resection of the stomach and incompleteness of vagotomy. The main clinical presentation of this condition is chronic abdominal pain, weight loss, diarrhea, gastrointestinal bleeding and fecal vomiting. The diagnostic workup should include barium enema, gastroscopy and sometimes colonoscopy and abdominal tomography for excluding and ruling out the possibility of malignant extraluminal disease. The historical approach of the treatment of this rare entity was 2-3-phased operations which included colostomy. However today, medical management has recently been recommended as the first-line therapy, with parenteral and enteral support treatments. The preferred surgical approach is single-stage gastrocolic resection and anastomosis and this has been favored to minimize mortality.
\end{abstract}

\section{Introduction}

Gastrojejunocolic fistula (GJF) is a rare and late complication of the surgical technique of gastrectomy and gastrojejunostomy applied for recurrent peptic ulcer disease.

Generally GJF is considered to be induced by a stomal ulcer due to inadequate gastric resection, incompleteness of vagotomy and long afferent loop $[1,2]$. The most frequent symptoms of such a fistula are upper abdominal pain, severe weight loss, diarrhea, gastrointestinal bleeding and sometimes fecal vomiting [3]. Physical examination and/or laboratory studies reveal malnutrition. The diagnosis is most reliably and frequently made by barium enema and gastroscopy $[4,5]$. 
In the management of the GJF, the historical approach was 2-3-phased operations which included colostomy in order to ameliorate the nutritional status and minimize the mortality of the patient [4-7]. However today, because of improved parenteral and enteral support treatments and the developments in intensive care conditions, single-stage procedures can be applied and these have been favored to minimize mortality $[2,8]$.

Although the occurence of GJF has decreased remarkably as a result of better medical treatment, the modern management of this condition and the generally accepted surgical treatment strategies must be discussed.

\section{Case Report}

A 51-year-old man was admitted to the surgical unit of Yuksek Ihtisas Hospital in October 2006 with complaints of chronic diarrhea, epigastric pain and discomfort, severe weight loss and weakness. His medical history was marked by a gastroenterostomy due to duodenal ulcer disease in 1992. The laboratory data on admission revealed hypoproteinemia and hypoalbuminemia. The patient was cachectic and dehydrated. At physical examination, liquidated intestine loops were sensed in the epigastrium and upper right quadrant. Laboratory parameters revealed serious electrolyte imbalance. A complete blood count revealed a hemoglobin level of $10.3 \mathrm{~g} / \mathrm{dl}$ (normal range 10.0-16.0) and an albumin level of $2.1 \mathrm{~g} / \mathrm{dl}$ (normal range 3.4-4.8) In the electrolyte values, the blood sodium level was $132 \mathrm{mEq} / \mathrm{l}$ (normal range 135-150) and the blood potassium level was $3.3 \mathrm{mEq} / \mathrm{l}$ (normal range 3.55.5). No disorder was determined in the other parameters. Posterior-anterior thorax X-ray film was unremarkable. Upper gastrointestinal series assessment confirmed the passage between the corpus of the stomach and the proximal jejunum ( $\underline{\text { fig. } 1}$ ). Transabdominal ultrasonography and tomography showed liquidated small intestine with edema and liquid between the intestine loops (fig. 2). Gastroscopy showed a previous gastrojejunostomy and a fecaloid secretion from the anastomotic region of the lumen. Colonoscopy showed an ulcer mass obstructing the transverse colon lumen and preventing the endoscope from passing to the proximal region. Biopsies were taken from these areas for histological examination.

Histology revealed edematous mucosa and no malignancy was detected. Liquid-electrolyte imbalance and nutritional deficiences were resuscitated. Total parenteral nutrition (TPN) was started immediately and continued in the postoperative period till the albumin level returned to normal. After improving his state of malnutrition, the patient was taken into the operation room and an exploratory laparotomy was undertaken. At surgery, a retrocolic gastroenterostomy, gastrojejunocolic fistula and incompleteness of vagotomy were identified (fig. 3 ). A radical en bloc resection was performed involving a subtotal gastrectomy, posterior vagectomy, partial transverse colon and jejunum resection. Restoration of bowel continuity was applied by gastroenterostomy, jejunojejunostomy and colocolostomy. Recovery was uneventful and the patient remained well at follow-up; once his general status had improved, he was taken into the normal service. Histology of the resected specimen revealed superficial ischemic changes and chronic inflammation.

\section{Discussion}

Today, the surgical operation necessity for peptic ulcer disease has decreased dramatically. Especially developments in the medical treatments with the usage of $\mathrm{H} 2$ receptor blockers, proton pump inhibitors and the eradication regimes for Helicobacter pylori play an active role. So the incidence rate of these fistulas has been remarkably lowered because of the decrease in surgery [1]. Since fistula formation needs a 20-30-year latent period after the initial surgery, this severe and rare complication may occur presently. Therefore, GJF is still an important and current complication $[2,3,9]$.

The symptoms of GJF are diarrhea with lienteric stools, epigastric pain and discomfort, gastrointestinal bleeding, feculent eructation, fecal vomiting, weight loss and weakness. Anemia, leukocytosis, electrolyte disturbances and hypoalbuminemia are common laboratory findings [10]. 
The most frequently used diagnostic tools are barium enema and endoscopy $[1,11-$ 13]. The success rate of barium enema in correctly diagnosing the fistula is approximately $95-100 \%[10,12,13]$. However, its determination success in the phenomenons which are related to malignancy is low [14]. Computerized axial tomography is used to determine this complex fistula and to exlude extraluminal diseases that may define underlying etiology $[15,16]$. Recently, endoscopy and colonoscopy are frequently used separately or together $[2,10]$. Especially the fistula orifice can be followed by upper gastrointestinal system endoscopy and when necessary, preoperative histological confirmation can be done [17-19].

Because of the fading effect of the disease, in the postoperative period, careful clinical and laboratory evaluation must be carried out. As specified before, in a large majority of these patients, the physical examinations and laboratory studies reveal low to medium malnutrition, anemia and electrolyte imbalance. As soon as possible, this condition must be recovered preoperatively. Therefore, TPN or total enteral nutrition (TEN) should be administered in patients who are suffering from malnutrition and this treatment protocol should continue in the postoperative period. Because of the physiologic effects of TEN over TPN, it should be the first choice if a nasoenteric tube can be placed into the jejunum [20].

The surgical options and treatments of gastrocolic fistula/GJF have changed over the years. The historical approach was 2-3-staged operations even involving a preliminary diversion colostomy in order to ameliorate the nutritional status of the patient and to decrease mortality $[4,6,7]$. In the late 1930s, the three-staged procedures included (1) colostomy, (2) resection of the fistula and (3) colostomy closure [10]. Then two-staged operation was defined. This operation, which is known as Lahey's procedure, was very popular because it was done with lower morbidity and mortality [21]. In the 1960s, it was stated that single-stage procedures could be applied [21,22]. Today, because of the parenteral and enteral support treatments and the developments in intensive care conditions, one-stage resection can be applied, and the mortality rates are getting lower $[2,3,8,21,22]$.

In conclusion, as a result of developments of the agents used in peptic ulcer treatment, GJF incidence has decreased dramatically. In the ancient times, recovering the medical status and staged operations were applied in GJF treatment, but today one-stage resections are preferred if the general status of the patient is suitable. TPN or TEN should be administered in patients suffering from malnutrition. In the preoperative diagnosis stage, a possible malignancy must be eliminated. 


\begin{tabular}{r|l|l|l}
$\begin{array}{r}\text { Case Reports in } \\
\text { Gastroenterology }\end{array}$ & $\begin{array}{l}\text { Case Rep Gastroenterol 2010;4:173-177 } \\
\text { D0I: 10.1159/000314048 }\end{array}$ & Published online: May 19, 2010 & $\begin{array}{l}\text { @ 2010 S. Karger AG, Basel } \\
\text { ISSN 1662-0631 } \\
\text { www.karger.com/crg }\end{array}$ \\
\hline
\end{tabular}

Fig. 1. Upper gastrointestinal series assessment confirmed the passage between the corpus of the stomach and the proximal jejunum.

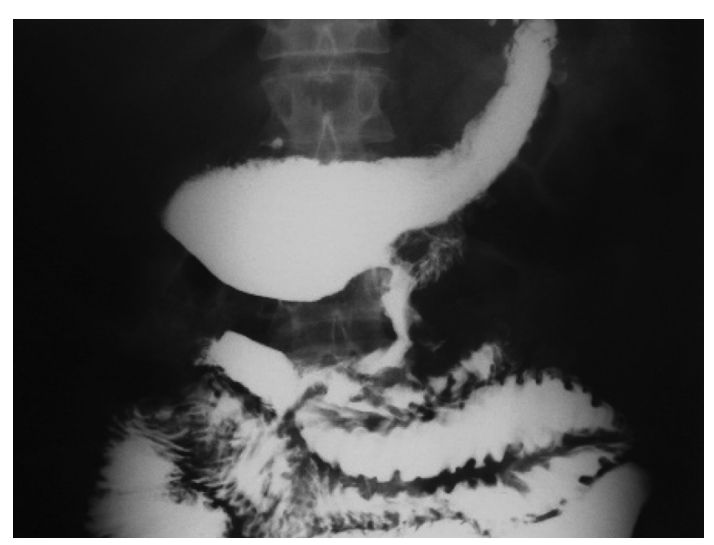

Fig. 2. Tomography showed liquidated small intestine with edema and liquid between the intestine loops.

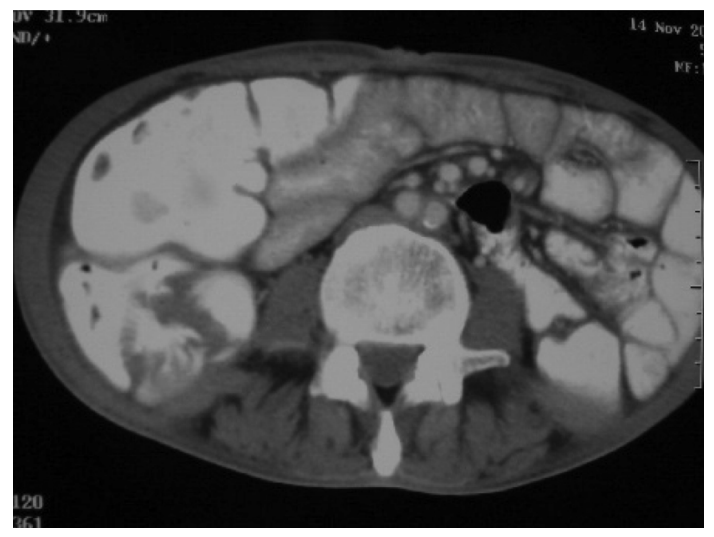

Fig. 3. At surgery, a retrocolic gastroenterostomy, gastrojejunocolic fistula and incomplete vagotomy were identified.

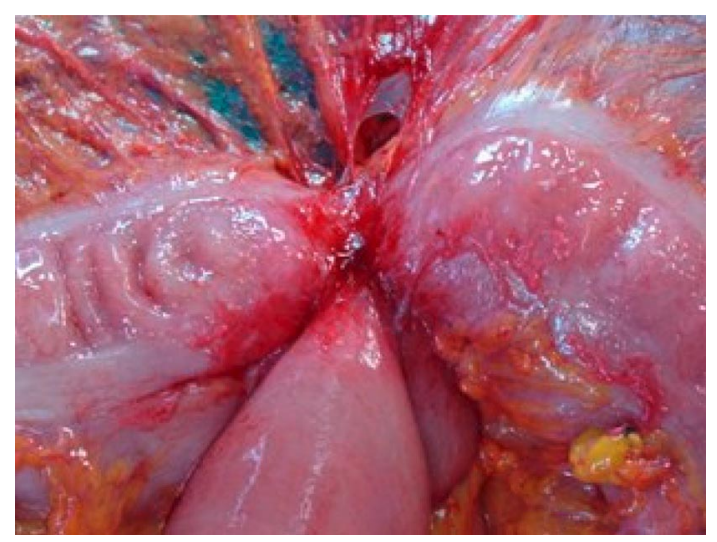




\section{References}

1 Damata G, Rahili A, Karimdjee-Soilihi B: Gastrojejunocolic fistula after gastric surgery for duodenal ulcer: case report. G Chir 2006;27:360-362.

2 Subramaniasivam N, Ananthakrishnan N, Kate V, Smile SR, Jagdish S, Srinivasan K: Gastrojejunocolic fistula following surgery for peptic ulcer. Trop Gastroenterol 1997;18:183-187.

-3 Chung DP, Li RS, Leong HT: Diagnosis and current management of gastrojejunocolic fistula. Hong Kong Med J 2001;7:439-441.

-4 Lowdon AG: Gastrojejunocolic fistula. Br J Surg 1953;41:113-128.

5 Thoeny RH, Hodgson JR, Scudamore HH: The roentgenologic diagnosis of gastrocolic and gastrojejunocolic fistulas. AJR Am J Roentgenol 1960;83:876-881.

6 Mathewson C Jr: Preliminary colostomy in the management of gastrocolic and gastrojejunocolic fistula. Ann Surg 1941;114:1004-1010.

7 Pfeiffer DB: The surgical treatment of gastrojejunocolic fistula. Surg Gynecol Obstet 1941;72:282-289.

8 Alhan E, Çalik A, Cinel A, Kucuktulu U: Complicated marginal ulcers after surgery for duodenal ulcer. Acta Chir Hung 1995-1996;35:77-85.

-9 Sorensen BM: Non malignant gastrointestinal shortcircuit (gastrojejunocolic fistula and gastroileostomy). Acta Chir Scand Suppl 1969;396:67-70.

10 Cody JH, Divincenti FC, Cowick DR, Mahanes JR: Gastrocolic and gastrojejunocolic fistula: Report of twelve cases and review of the literature. Ann Surg 1975;181:376-380.

11 Wilson RG, Wilson KS, Champion HR: Gastrojejunocolic fistula. J R Coll Surg Edinb 1973;18:227-230.

12 Wagtmans M, Kooy M, Snel P: Persistent diarrhoea in cholecystocolic and gastrocolic fistula after gastric surgery. Neth J Med 1993;43:218-221.

13 Ohta M, Konno H, Tanaka T, et al: Gastrojejunocolic fistula after gastrectomy with Billroth II reconstruction: report of a case. Surg Today 2002;32:367-370.

$\checkmark 14$ Forshaw MJ, Dastur JK, Murali K, Parker MC: Long-term survival from gastrocolic fistula secondary to adenocarcinoma of the transverse colon. World J Surg Oncol 2005;3:1-4.

15 Matsuo S, Eto T, Ohara O: Gastrocolic fistula originating from transverse colon cancer: report of a case and review of the Japanese literature. Surg Today 1994;24:1085-1089.

16 Lee WJ, Horton KM, Fishman EK: Gastrocolic fistula due to adenocarcinoma of the colon: simulation of primary gastric leiomyosarcoma on CT. Clin Imaging 1999;23:295-297.

17 Choi SW, Yarg JM, Kim SS: A case of combined gastrojejunal and gastrocolic fistula secondary to gastric cancer. J Korean Med Sci 1996;11:437-439.

18 Mimidis K, Papadopoulos V, Katsinelos P, et al: Gastrocolic fistula scondary to nonsteroidal anti-inflammatory drugs abuse in a cirrhotic patient. Rom J Gastroenterol 2004;13:39-41.

19 Tavenor T, Smith S, Sullivan S: Gastrocolic fistula. A review of 15 cases and an update of the literature. J Clin Gastroenterol 1993;16:189-191.

20 Zhang SY: Gastrojejunocolic fistula. Report of 5 cases. Zhanghua Wai Ke Za Zhi 1991;29:503-505.

21 Marshall S, Knud-Hansen J: Gastrojejunocolic and gastrocolic fistulas. Ann Surg 1957;145:770-782.

22 Barber KW, Waugh JK, Priestley JT: Operation in one stage for gastrojejunocolic fistula. Surg Clin North Am 1962;42:1443-1449.

Presented at the 23rd Turkish National Gastroenterology Congress, poster session, 4-7 November 2006, Istanbul, Turkey. 